\title{
A New Coastal Flood Forecasting System for the Netherlands
}

Topic: J. accurate hydrodynamics (e.g.: water levels and tides)

\section{Ir. Simone DE KLEERMAEKER, The Netherlands \\ Deltares}

Dr. Ir. Martin VERLAAN, The Netherlands

Deltares, TU Delft

Ing. Jan KROOS, The Netherlands

The Watermanagent Centre Netherlands of Rijkswaterstaat

Ir. Firmijn ZIJL, The Netherlands

Deltares

\section{INTRODUCTION}

The North Sea is one of the busiest seas in the world with dense ship traffic, fisheries, wind farming, recreation and many other activities. All these activities depend on the 'marine weather'. Accurate forecasts of waves, currents and sea level are crucial for operational management and for issuing warnings during extreme events.

The current operational forecasting system in the Netherlands has been operational since the 90 's. Over the last few years, a new generation forecasting system for the North Sea and the Dutch coastal waters has been developed. This involved both advanced model development as well as the set-up of an operational forecasting shell based on the FEWS (Flood Early Warning System) framework. From the start of the storm season on September $1^{\text {st }} 2012$, this new system will be used pre-operationally by Rijkswaterstaat (Dutch Ministry of Infrastructure and the Environment) and the Royal Netherlands Meteorological Institute, KNMI.

This paper gives an overview of the many developments that are needed for an accurate and reliable forecasting system. In addition to accurate and robust numerical models, one needs scheduling with backup options, quality control of observations, good interactive displays of many datasets, manual override for operators, and much more. The quality of the operational forecasts is as strong as the weakest link.

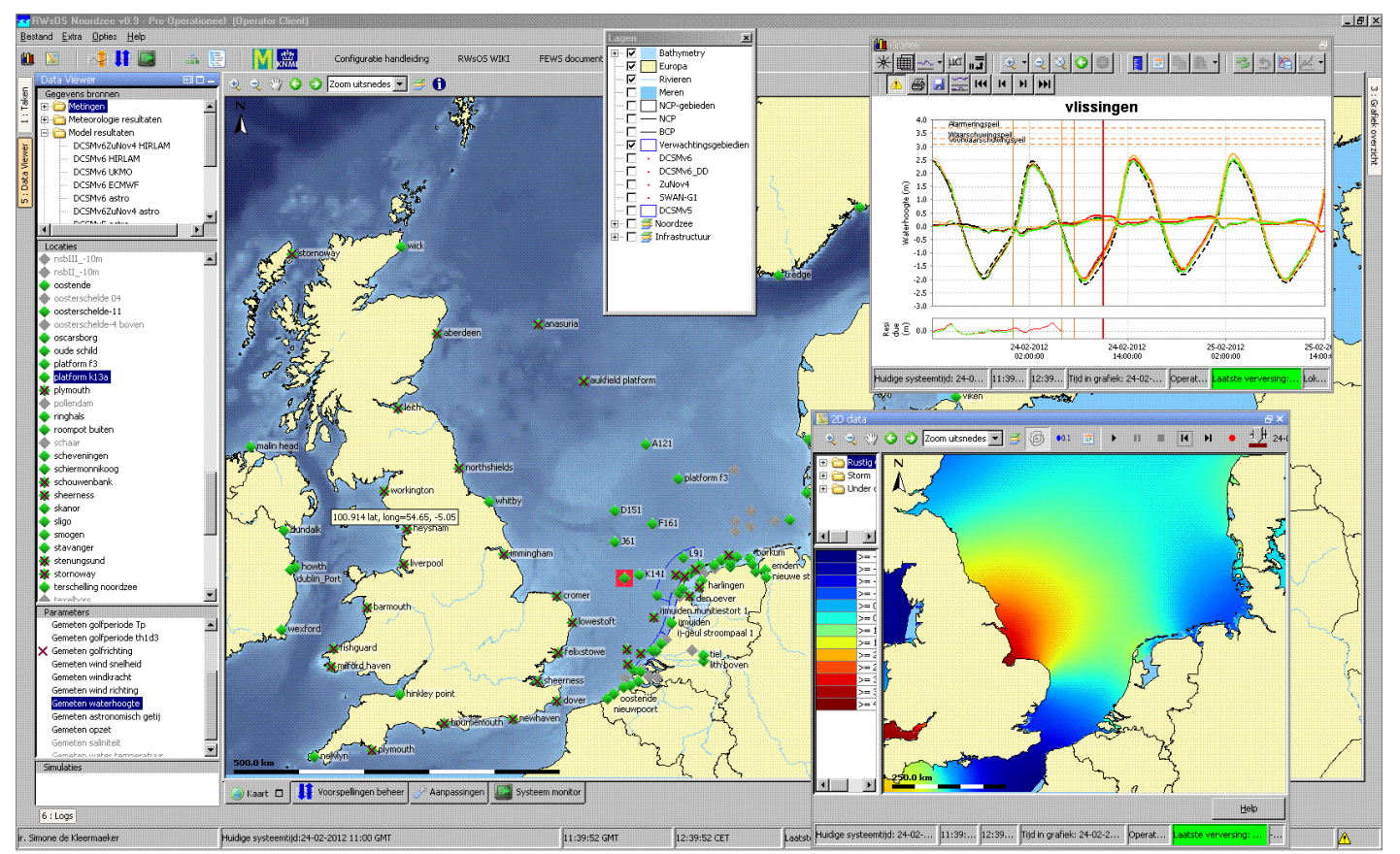

Figure 1: Screen shot of the operational system FEWS North Sea 


\section{OPERATIONAL SYSTEM}

In daily operations, the new FEWS North Sea system will be used for water management of Dutch coastal waters, e.g. to ensure maritime safety on busy shipping routes towards the large sea ports of Rotterdam, Antwerp and IJmuiden. The system also generates boundary conditions for fluvial models for the port of Rotterdam, the area of Dordrecht and the Scheldt estuary. During storm surges the forecasts will be used to issue warnings to coastal authorities, based on which measures can be taken.

The system is used to collect all necessary data and coordinate computations. Automated runs are scheduled 4 times a day, following the release of new meteorological forecasts from KNMI. Model output can be inspected and compared with observations, to assist forecasters to decide on appropriate warning levels. The distributed water level forecasts and warnings are based on model forecasts as well as on expert judgement of the forecasters. The system automatically synchronizes between multiple forecasters located in different offices or through mobile telecommunication. In case of a hardware failure, the forecast system automatically switches to a stand-by system.

The system is a major step forward both in accuracy of the model forecasts [Verlaan et. al, 2003] and in combining data from different sources into easily interpretable displays. It also assists the forecasters in cooperating between multiple offices of Rijkswaterstaat and KNMI.

\section{OBSERVATIONS AND METEOROLOGICAL FORCING CONDITIONS}

The operational system collects all data required to create the water level and wave forecasts. This includes observations of water level, wave data such as wave height, swell, period and direction, and also of air pressure, and wind speed and direction. Besides being used to monitor the current state of the North Sea and to identify possible deviations, observations are used for (real-time) data-assimilation.

\section{Automatic validation of water level observations}

All models, including data-assimilation (see the corresponding Sections below), are scheduled to run automatically and mostly unattended at regular intervals. Therefore, it is essential to apply a good automated quality control to the observations. As a backup procedure, the operators can overrule the automated QC and visually inspect the observations and discard invalid data

The automatic quality control is based on the following rules:

- Absolute limits, which provide a crude test useful only to identify very large errors.

- Earlier or later (high frequency) observations at the same location, to detect spikes.

- Comparison with observations at a nearby locations.

- Comparison with the harmonic tide as a first estimate, and

- Comparison with a model first-guess, which can be expected to show much less deviation from the observation than the harmonic tide.

Rejection of all observations which exceed one of the limits usually leads to excessive loss of good data or acceptance of erroneous data, depending on whether the limits are narrow or not. Therefore a sensible combination of the qualifications is needed. We have chosen to make an arithmetical, rather than a logical, combination of the qualifications into a qualification score.

\section{Numerical Meteorological forecasts}

Meteorological forecasts serve as one of the main forcings for the water level and wave models. The standard model suite uses forecasts from the HiRLAM Numerical Weather Prediction model provided operationally by KNMI, which has a forecast horizon of 48 hours. 


\section{Additional numerical meteorological forecasts}

For a reliable forecasting system, the possibility of a failure has to be considered. For this purpose additional computations are carried out with alternative meteorological forcing. These model results also gives the forecaster the possibility to assess sensitivities and expected magnitude of errors.

Alternative numerical meteorological forecasts are obtained from ECMWF and the UK Met Office (UKMO). UKMO is used for comparison and as a back-up of the HiRLAM model. This model has a horizon of 5 days. For the longer term forecast the ECMWF model is used, provided by the European Centre for Medium range Weather Forecasting. This model has a lower resolution but has a forecast horizon of 10 days. The lower resolution model DCSMv5 is run with the full range of the ECMWF EPS (Ensemble Prediction System) forecasts, to give an impression of the accuracy of the mid-range forecast.

\section{WATER LEVEL MODEL}

Part of the comprehensive upgrade of the operational forecasting system was the development of a new generation operational flood forecasting model for the Northwest European Shelf and North Sea. This Dutch Continental Shelf Model (DCSMv6) is a 2D (horizontal) application of SIMONA, the framework for hydrodynamic modelling of freesurface water systems used for Rijkswaterstaat modelling [SIMONA documentation]. Compared to the previous version (v5) internal tide generation has been included, while the cell sizes of the spherical grid have been decreased substantially to $1.5^{\prime}\left(1 / 40^{\circ}\right)$ in east-west direction and $1.0^{\prime}\left(1 / 60^{\circ}\right)$ in north-south direction, leading to more than $10^{6}$ grid cells. To reduce the computational time required to provide forecasts, parallel computing has been applied. With a computational time step of 2 minutes, a 1 day simulation takes approximately 5 minutes on a dual hexacore machine.

The spatial extent of the model has been increased, in order to ensure that the open boundaries are located in deeper water. This makes it possible to use harmonic boundary forcing derived from global tidal models. Furthermore, wind setup in deep water can safely be neglected, whereas the time- and space varying effect of local pressure (the so-called inverse barometer effect) is added to the water level variation along the open boundary. Tidal water levels at the open boundaries are specified in the frequency domain, i.e. the amplitudes and phases of a number of tidal constituents are specified. The tidal conditions of the eight main diurnal and semi-diurnal constituents have been derived from the GOT00.2 global ocean tide model. Sixteen smaller diurnal and semi-diurnal constituents have been derived by means of the admittance method. Additionally, the solar annual constituent Sa is specified, based on satellite altimeter data from the Topex-Poseidon and Jason missions.

Well distributed water level observations at more than 100 coastal tide gauge locations have been used for a thorough calibration and validation of the shelf areas. Automatic calibration methods available in the open source data assimilation toolbox OpenDA [OpenDA documentation] were applied to optimize over 200 control parameters for bathymetry and bed friction. Furthermore, a series of Goodness-of-Fit (GoF) measures (in frequency and time domain) was determined to quantify the quality of various aspects of the water level representation per sub-region and for the model as such. This structured approach has resulted in a significant reduction in errors at stations along the Dutch coast. The average RMSE as calculated for the year 2007 has reduced (compared to the previous operational model) from $13.1 \mathrm{~cm}$ to $7.8 \mathrm{~cm}$, which implies a reduction in error of $40 \%$. Considering tide only, the reduction is an impressive $60 \%$ (from $9.5 \mathrm{~cm}$ to $3.7 \mathrm{~cm}$ ).

\section{Data-assimilation}

To further improve the accuracy of the forecasts for lead times up to around 15 hours, a steady-state Kalman filter has been developed. For this purpose the generic open source data assimilation toolbox OpenDA was integrated into the SIMONA modelling software. Observations of sea level (with a 10-minute interval) from over 30 tide gauge stations are 
assimilated every forecast cycle. In hindcast mode, in which historic observations are available for assimilation, the average RMSE along the Dutch coast for the year 2007 is $3.5 \mathrm{~cm}$. As this development is still ongoing, the improvement of the forecast accuracy for a range of lead-time intervals has yet to be quantified.

\section{WAVE MODEL}

The operational wave model NEDWAM from KNMI will be replaced by the newly developed SWAN-North Sea wave model [SWAN documentation]. This SWAN model covers a larger area and has a higher resolution than the NEDWAM model, both to increase the accuracy near shore. The model covers a large part of the northwest continental shelf and consists of two grids. The larger grid computes boundary conditions for the high resolution grid of the southern North Sea. The model is driven by HiRLAM wind fields, spectral wave conditions from ECMWF on the boundaries and water level and current fields from the DCSMv6 model.

A preliminary version gave $10 \%$ bias (18\% scatter) in wave height, and $0 \%$ (15\% scatter) in period. This results is already comparable to NEDWAM, and we see room for further improvement. The final calibration and validation is still ongoing. Data-assimilation for this model is being developed in the framework of the EU-project MyWave [MyWave].

\section{DISPLAY, INTERPRETATION, AND DISTRIBUTION OF DATA}

A crucial step in the operational process is to interpret and judge the available model results and observations (see Figure 3). Operational forecast often have to be made under time pressure, which combined with the large amount of different datasets can easily lead to a mistake, that can have serious consequences. Therefore the data should be presented in a way that is easy to interpret and consistent.

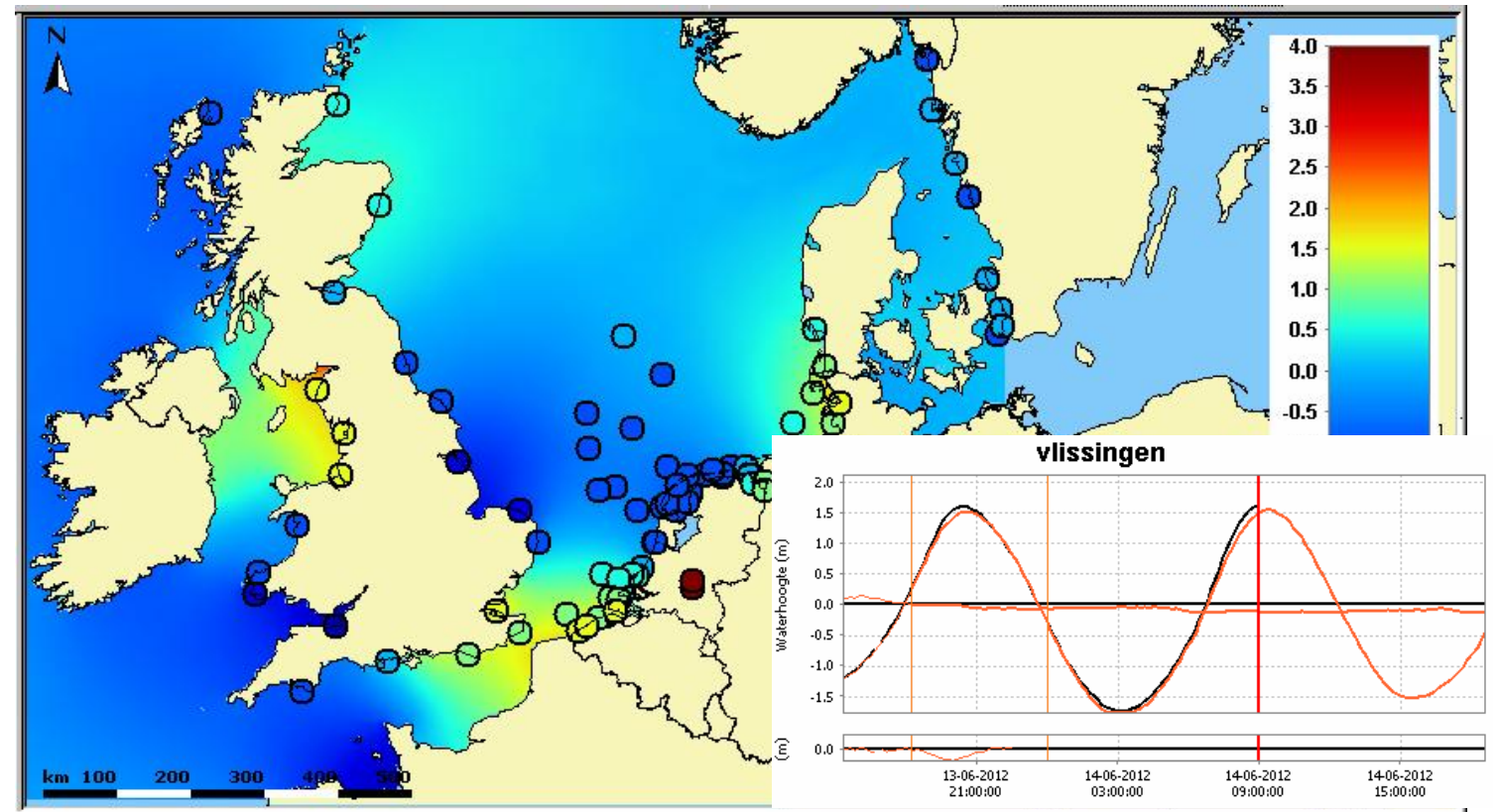

Figure 2: Spatial display of the Southern North Sea with water level [m] model results, combined with observations in circles. Overlay shows time series for one location with water level $[\mathrm{m}]$ model result and residue combined with the local observation (in black).

\section{Predefined plots}

In addition to the possibility to explore all the available data freely and manually create arbitrary combinations of data in a plot, a number of predefined plots were created. One set of predefined plots show the observed and forecasted sea level for a number of main locations. The difference between the model results and the observations, i.e. the residue, is 
calculated and shown as well. The forecaster can turn off part of the data, offering him some freedom combined with the consistency of how the data is displayed.

Combining different observations and results into one graph has more benefits than the obvious ease of overview. It can prevent mistakes made due to difference in presentation, such as scales or units, colour schemes and time zones. By organizing all data and pre- and post-processing in one system, the forecaster is assured of consistent information. The FEWS system is available to partners like KNMI, which means that both the hydrological and the meteorological forecasters use exactly the same displays. This makes discussions over the phone easier and less sensitive to communication errors.

\section{Comparison of multiple forecasts}

The models have a forecast horizon of minimum 2 days and are run every 6 hours. Therefore, the same window in time is forecasted several times. The variability between subsequent forecasts can give the forecaster information on changes and the stability of the model results and therefore the trustworthiness of the forecast. For this, we have developed a spatial display in which spatial data of several forecast for the same timeframe can be compared to each other. The thumbnails (see Figure 3 ) can be quickly inspected to locate differences. Each thumbnail can be selected to display the data in more detail in the main window.

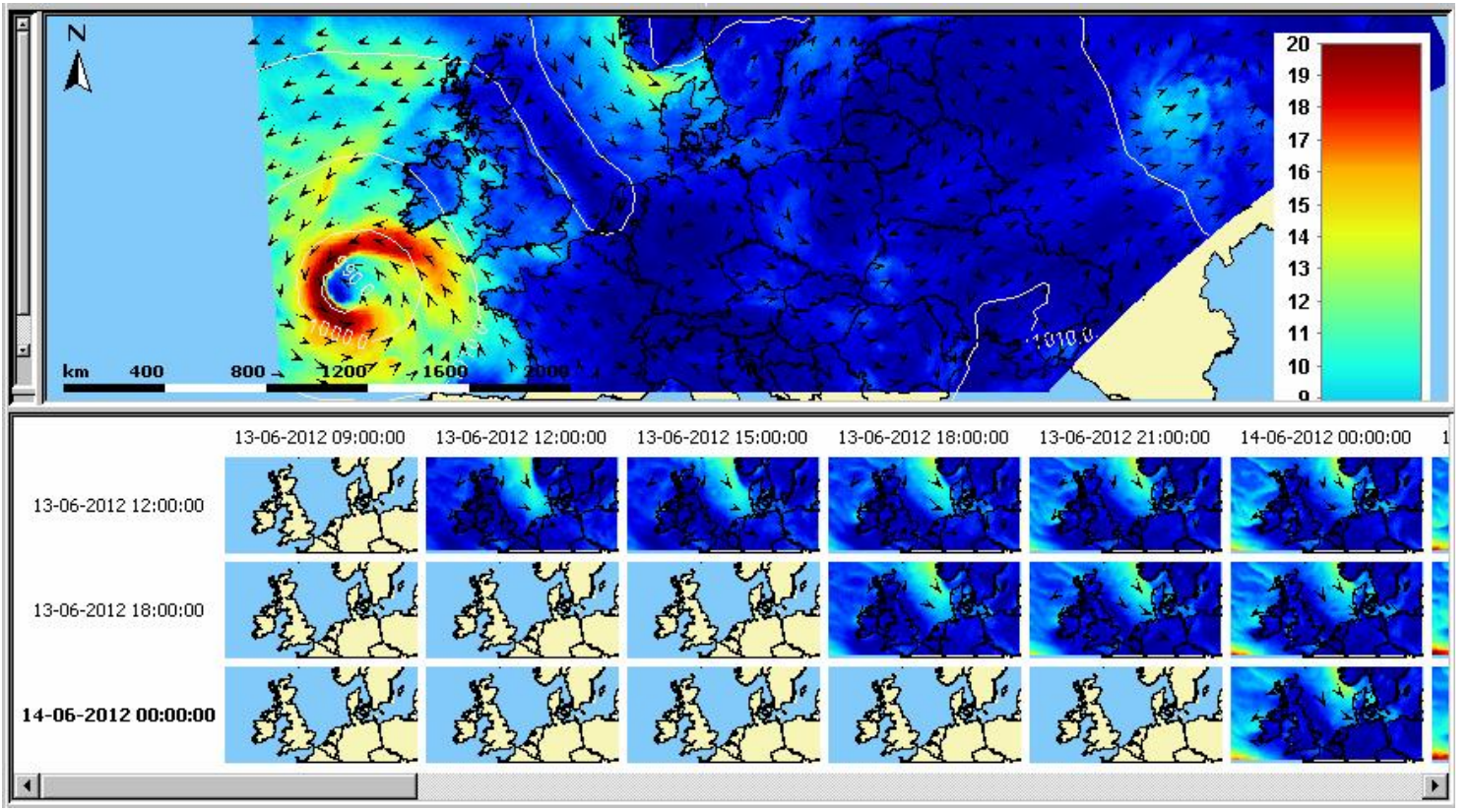

Figure 3: Three meteorological models run six hours apart. Thumbnails every three hours.

\section{Generating the official forecast}

A composite water level forecast is generated automatically. For the forecast horizon of $0-12$ hours the model with data-assimilation is used. This is extended to 48 hours using the model results with Hirlam forcing. The model results with ECMWF forcing are used to further extend the forecast horizon to 10 days. If any model is not functioning properly, the model next in hierarchy is automatically used as a fall back option.

The forecaster adjusts this composite forecast where necessary based on his expert judgement. He is assisted by the FEWS system allowing easy data analysis and discussions with the meteorological expert.

\section{Distribution of warnings}

The final step in the operational process is to distribute the official forecast and appropriate warnings. The official forecast is distributed using the FEWS North Sea system towards the 
National database and internet. By generating the warning messages within the same system, mistakes in data input can be prevented.

\section{CONCLUSION}

Generating a forecast in the operational environment is based on more than a state of the art models alone. The entire work process from import and quality control of observations, via the running of the models and the interpretation of the results up to the distribution of the final warnings has to function properly. The weakest link in this chain of actions determines the strength of early warning system Therefore, the operational system should be judged as a whole, considering both accuracy and reliability.

\section{REFERENCES}

FEWS documentation, accessed June $3^{\text {rd }}, 2012$ http://publicwiki.deltares.nl/display/FEWSDOC/ MyWave, accessed June $15^{\text {th }}, 2012$. http://cordis.europa.eu/; search for mywave OpenDA documentation, accessed June $3^{\text {rd }}, 2012$. http://www.openda.org/joomla/index.php SIMONA documentation (in Dutch), accessed June $3^{\text {rd }}, 2012$. http://www.helpdeskwater.nl/onderwerpen/applicaties-modellen/water_en_ruimte/simona/ SWAN manual. 2011. "User Manual", accessed June $3^{\text {rd }}$ 2012. http://www.swan.tudelft.nl/ $\mathrm{M}$. Verlaan, A. Zijderveld, H. De Vries, J. Kroos, Operational storm surge forecasting in the Netherlands: developments in the last decade, Phil. Trans. R. Soc. A (2005) 363, 1441-1453, doi:10.1098/rsta.2005.1578

\section{BIOGRAPHIES}

Simone DE KLEERMAEKER is senior advisor in the Marine and Coastal Systems Unit of Deltares. She has more than 10 years of experience with hydrodynamic modelling of both Marine and Coastal as well as Industrial systems, both as a project engineer and project leader. She applies these models in projects such as Serious Gaming, FEWS installations and calibration using tools such as OpenDA. She has a background in fluid dynamics, based upon her graduation in Technical Mechanics with specialisation on fluid dynamics.

Martin VERLAAN is a senior data-assimilation specialist at Deltares and assistant professor at Delft Technical University. He has almost 20 years of experience in data-assimilation and storm surge modelling. Current research topics include data-assimilation for wave models and quantification of the value of observations in data-assimilation. He is one of the lead developers of OpenDA and secretary of the OpenDA association. He is also a senior member of the Storm Surge Warning Service of the Netherlands (SVSD).

Jan KROOS is the teamleader of the Storm Surge Warning Service of the Netherlands (SVSD). The SVSD is a part of the WaterManagement Centre of the Netherlands (WMCN) of Rijkswaterstaat. He has over 30 years of experience in operational storm surge forecasting. $\mathrm{He}$ is also a member of the national flood coordination committee in the Netherlands, that gives advice to the appropriate national, regional and local authorities in the case that severe storms or extreme river flow that would threaten to flood parts of the Netherlands.

Firmijn ZIJL is senior reasearcher/advisor at the Marine and Coastal Systems Unit of Deltares, with a background in computational fluid mechanics. He has almost 10 years of experience with hydrodynamic modelling of free surface flows. He was responsible for the development of models in complex tidal systems (e.g. the Singapore region and the Northwest European Shelf) as well as 3D baroclinic models of weakly dynamic, stratified seas and deep lakes (e.g. the Sea of Marmara and various reservoirs in the Netherlands and Singapore). 